\title{
Sound waves in two-dimensional ducts with sinusoidal walls
}

\author{
Ali Hasan Nayfeh
}

Department of Engineering Science and Mechanics, Virginia Polytechnic Institute and State University, Blacksburg, Virginia 24061

(Received 7 March 1974; revised 21 April 1974)

\begin{abstract}
The method of multiple scales is used to analyze the wave propagation in two-dimensional hard-walled ducts with sinusoidal walls. For traveling waves, resonance occurs whenever the wall wavenumber is equal to the difference of the wavenumbers of any two duct acoustic modes. The results show that neither of these resonating modes could occur without strongly generating the other.
\end{abstract}

Subject Classification: $20.45,20.50$.

\section{INTRODUCTION}

We consider acoustic waves propagating in an inviscid, nonconducting, perfect gas confined in a two-dimensional hard-walled duct having a nonuniform cross section. We assume the walls to have weak sinusoidal undulations. We make lengths, velocities, and time dimensionless using the average width of the duct $d$, the undisturbed speed of sound $c$, and the characteristic time $d / c$. In dimensionless quantities, the walls of the duct are located by

$$
\begin{array}{ll}
y=\epsilon \sin k_{w} x, & \text { lower wall, } \\
y=1+\epsilon \sin \left(k_{w} x+\theta\right), & \text { upper wall, }
\end{array}
$$

where $\epsilon$ is a small dimensionless parameter characterizing the weakness of the wall undulations, $k_{w}$ is the wavenumber of the undulations, and $\theta$ is the phase difference between the undulations of the two walls.

Since the gas is assumed to be inviscid and nonconducting, its irrotational motion can be described by a dimensionless potential function $\tilde{\phi}(x, y, t)$. For linear motions and harmonic time variations of the form $\bar{\phi}(x$, $y, t)=\phi(x, y) \exp (-i \omega t), \phi$ satisfies

$$
\nabla^{2} \phi+\omega^{2} \phi=0 \text {, }
$$

where $\omega$ is the dimensionless frequency of oscillation. For an inviscid fluid and a hard wall, the flow is tangential to the walls; that is,

$$
\begin{array}{ll}
\phi_{y}=\epsilon \phi_{x} k_{w} \cos k_{w} x, & \text { at } y=\epsilon \sin k_{w} x, \\
\phi_{y}=\epsilon \phi_{x} k_{w} \cos \left(k_{w} x+\theta\right), & \text { at } y=1+\epsilon \sin \left(k_{w} x+\theta\right) .
\end{array}
$$

Straightforward expansions of the form $\phi_{0}+\epsilon \phi_{1}$ were obtained for the solutions of this problem by Isakovitch ${ }^{1}$ for the case of a waveguide with only one undulating wall, by Samuels ${ }^{2}$ for the case of a waveguide with inphase wall undulations (i.e., $\theta=0$ ), and by Salant ${ }^{3}$ for the above general problem. Unfortunately, all the above expansions are not uniform because the correction $\epsilon \phi_{1}$ dominates the first term $\phi_{0}$ for frequencies near what Isakovitch, Samuels, and Salant call the resonant frequencies. In this paper, we determine a uniform expansion by using the method of multiple scales. ${ }^{4}$ Before determining this uniform expansion, we carry out a straightforward expansion in the next section to exhibit the nonuniformity.

\section{A STRAIGHTFORWARD EXPANSION}

In this section, we follow Salant and seek an expansion of the form

$$
\phi(x, y)=\phi_{0}(x, y)+\epsilon \phi_{1}(x, y)+\cdots .
$$

Substituting Eq. 5 into Eqs. 2-4, transferring the boundary conditions from $y=\epsilon \sin k_{w} x$ and $y=1+\epsilon \sin \left(k_{w} x\right.$ $+\theta)$ to $y=0$ and $y=1$ by developing $\phi_{x}$ and $\phi_{y}$ in Taylor series expansions, expanding for small $\epsilon$, and equating coefficients of like powers of $\epsilon$, we obtain

Order $\epsilon^{0}$

$$
\begin{aligned}
& \nabla^{2} \phi_{0}+\omega^{2} \phi_{0}=0, \\
& \phi_{0 y}=0, \text { at } y=0, \\
& \phi_{0 y}=0, \text { at } y=1 ;
\end{aligned}
$$

Order $\epsilon$

$$
\begin{aligned}
& \nabla^{2} \phi_{1}+\omega^{2} \phi_{1}=0, \\
& \phi_{1 y}=\phi_{0 x} k_{w} \cos k_{w} x-\phi_{0 y y} \sin k_{w} x \text {, at } y=0, \\
& \phi_{1 y}=\phi_{0 x} k_{w} \cos \left(k_{w} x+\theta\right)-\phi_{0 y y} \sin \left(k_{w} x+\theta\right), \text { at } y=1 .
\end{aligned}
$$

The solution of Eqs. 6-8 is taken to be

$$
\phi_{0}=A \cos n \pi y \exp \left(i k_{n} x\right),
$$

where

$$
k_{n}^{2}=\omega^{2}-n^{2} \pi^{2}, n=0,1,2, \cdots .
$$

For traveling waves, $n \pi$ must be less than $\omega$.

Substituting for $\phi_{0}$ into Eqs. 10 and 11, we have

$$
\begin{aligned}
& \phi_{1 y}= \frac{1}{2} i A\left(k_{n} k_{w}-n^{2} \pi^{2}\right) \exp \left[i\left(k_{n}+k_{w}\right) x\right] \\
&+\frac{1}{2} i A\left(k_{n} k_{w}+n^{2} \pi^{2}\right) \exp \left[i\left(k_{n}-k_{w}\right) x\right], \text { at } y=0, \\
& \phi_{1 y}= \frac{1}{2} i A\left(k_{n} k_{w}-n^{2} \pi^{2}\right) \cos n \pi \exp \left[i\left(k_{n}+k_{w}\right) x+i \theta\right] \\
&+\frac{1}{2} i A\left(k_{n} k_{w}+n^{2} \pi^{2}\right) \cos n \pi \exp \left[i\left(k_{n}-k_{w}\right) x-i \theta\right], \\
& \text { at } y=1 .
\end{aligned}
$$

We seek a particular solution to Eqs. 9, 14, and 15 of the form

$$
\begin{aligned}
\phi_{1}= & \frac{1}{2} i A\left(k_{n} k_{w}-n^{2} \pi^{2}\right) \Phi_{1}(y) \exp \left[i\left(k_{n}+k_{w}\right) x\right] \\
& +\frac{1}{2} i A\left(k_{n} k_{w}+n^{2} \pi^{2}\right) \Phi_{2}(y) \exp \left[i\left(k_{n}-k_{w}\right) x\right] .
\end{aligned}
$$

Substituting Eq. 16 into Eqs. 9, 14, and 15 and equating the coefficients of $\exp \left[i\left(k_{n} \pm k_{w}\right) x\right]$, we obtain 


$$
\begin{aligned}
& \Phi_{1}^{\prime \prime}+\alpha_{1}^{2} \Phi_{1}=0, \quad \alpha_{1}^{2}=\omega^{2}-\left(k_{n}+k_{w}\right)^{2}, \\
& \Phi_{1}^{\prime}(0)=1, \\
& \Phi_{1}^{\prime}(1)=\cos n \pi \exp (i \theta) ; \\
& \Phi_{2}^{\prime \prime}+\alpha_{2}^{2} \Phi_{2}=0, \quad \alpha_{2}^{2}=\omega^{2}-\left(k_{n}-k_{w}\right)^{2}, \\
& \Phi_{2}^{\prime}(0)=1, \\
& \Phi_{2}^{\prime}(1)=\cos n \pi \exp (-i \theta) .
\end{aligned}
$$

The solutions of Eqs. 17 and 18 are

$$
\begin{aligned}
\Phi_{j}= & \left(\alpha_{j} \sin \alpha_{j}\right)^{-1}\left\{\sin \alpha_{j} \sin \alpha_{j} y+\left[\cos \alpha_{j}-\cos n \pi\right.\right. \\
& \left.\times \exp (-i \theta \cos \pi j)] \cos \alpha_{j} y\right\}, j=1 \text { and } 2 .
\end{aligned}
$$

The present expansion is in agreement with that obtained by Salant.

The functions $\Phi_{j} \rightarrow \infty$, and hence, $\phi_{1} \rightarrow \infty$ as $\alpha_{j} \rightarrow m \pi$, which corresponds to the resonant frequencies

$$
\omega_{r}^{2}=\left(k_{n} \pm k_{w}\right)^{2}+m^{2} \pi^{2} \text {, with integer } m .
$$

Hence, the above straightforward expansion is not valid when $\omega \rightarrow \omega_{r}$. An expansion valid near the resonant frequencies is obtained in the next section by using the method of multiple scales. Since $k_{m}^{2}=\omega^{2}-m^{2} \pi^{2}$ from Eq. 13, the resonant frequencies occur whenever

$$
k_{w}=k_{n} \pm k_{m} \text {. }
$$

Note that, for the special case $\theta=0, m$ takes on all odd values when $n$ is even and $m$ takes on all even values when $n$ is odd. For the special case $\theta=\pi, m-n$ is even. For $\theta \neq 0$ and $\pi, m$ takes on all integer values. The resonant case $k_{w}=k_{n}+k_{m}$ occurs only for standing waves, while the other case occurs for both standing and traveling waves. In this paper, we consider the traveling case only.

\section{EXPANSIONS VALID NEAR RESONANT FREQUENCIES}

In this section, we determine uniform expansions for the resonant case $k_{w} \approx k_{n}-k_{m}$. To this end, we seek asymptotic expansions to the solutions of Eqs. 2-4 of the form

$$
\phi(x, y)=\phi_{0}\left(x_{0}, x_{1}, y\right)+\epsilon \phi_{1}\left(x_{0}, x_{1}, y\right)+\cdots,
$$

where $x_{0}=x$ is a fast scale characterizing the wavelengths of the acoustic waves and $x_{1}=\epsilon x$ is a slow scale characterizing the amplitude and phase modulations due to the resonance. Substituting Eq. 22 into Eqs. 2-4 and equating coefficients of like powers of $\epsilon$, we obtain

Order $\epsilon^{0}$

$$
\begin{aligned}
& \frac{\partial^{2} \phi_{0}}{\partial x_{0}^{2}}+\frac{\partial^{2} \phi_{0}}{\partial y^{2}}+\omega^{2} \phi_{0}=0, \\
& \phi_{0 y}=0, \text { at } y=0, \\
& \phi_{0 y}=0, \text { at } y=1 ;
\end{aligned}
$$

Order $\epsilon$

$$
\frac{\partial^{2} \phi_{1}}{\partial x_{0}^{2}}+\frac{\partial^{2} \phi_{1}}{\partial y^{2}}+\omega^{2} \phi_{1}=-2 \frac{\partial^{2} \phi_{0}}{\partial x_{0} \partial x_{1}},
$$

$$
\begin{array}{r}
\frac{\partial \phi_{1}}{\partial y}=k_{w} \frac{\partial \phi_{0}}{\partial x_{0}} \cos k_{w} x_{0}-\frac{\partial^{2} \phi_{0}}{\partial y^{2}} \sin k_{w} x_{0}, \text { at } y=0, \\
\frac{\partial \phi_{1}}{\partial y}=k_{w} \frac{\partial \phi_{0}}{\partial x_{0}} \cos \left(k_{w} x_{0}+\theta\right)-\frac{\partial^{2} \phi_{0}}{\partial y^{2}} \sin \left(k_{w} x_{0}+\theta\right), \\
\text { at } y=1 .
\end{array}
$$

The solution of Eqs. 23-25 is taken to contain the two interacting modes; that is,

$$
\phi_{0}=A_{m}\left(x_{1}\right) \cos m \pi y \exp \left(i k_{m} x_{0}\right)+A_{n}\left(x_{1}\right) \cos n \pi y \exp \left(i k_{n} x_{0}\right),
$$

where $A_{m}\left(x_{1}\right)$ and $A_{n}\left(x_{1}\right)$ are still undetermined at this level of approximation; they are determined at the next level of approximation. Substituting for $\phi_{0}$ from Eq. 29 into Eqs. 26-28, we obtain

$$
\begin{aligned}
& \frac{\partial^{2} \phi_{1}}{\partial x_{0}^{2}}+\frac{\partial^{2} \phi_{1}}{\partial y^{2}}+\omega^{2} \phi_{1}=-2 i k_{m} A_{m}^{\prime} \cos m \pi y \exp \left(i k_{m} x_{0}\right) \\
& -2 i k_{n} A_{n}^{\prime} \cos n \pi y \exp \left(i k_{n} x_{0}\right), \\
& \phi_{1 y}=\frac{1}{2} i \sum_{j=m, n} A_{j}\left(k_{j} k_{w}-j^{2} \pi^{2}\right) \exp \left[i\left(k_{j}+k_{w}\right) x_{0}\right] \\
& +\frac{1}{2} i \sum_{j=m, n} A_{j}\left(k_{j} k_{w}+j^{2} \pi^{2}\right) \exp \left[i\left(k_{j}-k_{w}\right) x_{0}\right], \text { at } y=0 \text {, } \\
& \phi_{1 y}=\frac{1}{2} i \sum_{j=m, n} A_{j}\left(k_{j} k_{w}-j^{2} \pi^{2}\right) \cos j \pi \exp \left[i\left(k_{j}+k_{w}\right) x_{0}+i \theta\right] \\
& +\frac{1}{2} i \sum_{j=m, n} A_{j}\left(k_{j} k_{w}+j^{2} \pi^{2}\right) \cos j \pi \exp \left[i\left(k_{j}-k_{w}\right) x_{0}-i \theta\right], \\
& \text { at } y=1 \text {, }
\end{aligned}
$$

where primes denote differentiation with respect to $x_{1}$.

Equations 30-32 will have a finite solution, and hence the resulting expansion is uniform if, and only if, a socalled solvability condition is satisfied. To determine this solvability condition and carry out the expansion further, we express the nearness of $k_{w}$ to $k_{n}-k_{m}$ by introducing the detuning parameter $\sigma$ according to

$$
k_{w}=k_{n}-k_{m}+\epsilon \sigma, \sigma=0(1),
$$

and express $\left(k_{n}-k_{w}\right) x_{0}$ and $\left(k_{m}+k_{w}\right) x_{0}$ as

$$
\begin{aligned}
& \left(k_{n}-k_{w}\right) x_{0}=k_{m} x_{0}-\sigma x_{1}, \\
& \left(k_{m}+k_{w}\right) x_{0}=k_{n} x_{0}+\sigma x_{1} .
\end{aligned}
$$

To determine the solvability condition for Eqs. 30-32, we seek a particular solution of the form

$$
\phi_{1}=i \Phi_{m}(y) \exp \left(i k_{m} x_{0}\right)+i \Phi_{n}(y) \exp \left(i k_{n} x_{0}\right) .
$$

Substituting Eq. 35 into Eqs. 30-32, using Eqs. 34, and equating the coefficients of $\exp \left(i k_{m} x_{0}\right)$ and $\exp \left(i k_{n} x_{0}\right)$ on both sides, we obtain

$$
\begin{aligned}
& \Phi_{m}^{\prime \prime}+m^{2} \pi^{2} \Phi_{m}=-2 k_{m} A_{m}^{\prime} \cos m \pi y, \\
& \Phi_{m}^{\prime}(0)=\frac{1}{2} A_{n}\left(k_{n} k_{w}+n^{2} \pi^{2}\right) \exp \left(-i \sigma x_{1}\right), \\
& \Phi_{m}^{\prime}(1)=\frac{1}{2} A_{n}\left(k_{n} k_{w}+n^{2} \pi^{2}\right) \cos n \pi \exp \left[-i\left(\sigma x_{1}+\theta\right)\right], \\
& \Phi_{n}^{\prime \prime}+n^{2} \pi^{2} \Phi_{n}=-2 k_{n} A_{n}^{\prime} \cos n \pi y, \\
& \Phi_{n}^{\prime}(0)=\frac{1}{2} A_{m}\left(k_{m} k_{w}-m^{2} \pi^{2}\right) \exp \left(i \sigma x_{1}\right), \\
& \Phi_{n}^{\prime}(1)=\frac{1}{2} A_{m}\left(k_{m} k_{w}-m^{2} \pi^{2}\right) \cos m \pi \exp \left[i\left(\sigma x_{1}+\theta\right)\right] .
\end{aligned}
$$

The general solution of Eq. 36 is 


$$
\Phi_{m}=c_{1} \cos m \pi y+c_{2} \sin m \pi y-\left(k_{m} / m \pi\right) y A_{m}^{\prime} \sin m \pi y .
$$

Substituting Eq. 42 into Eqs. 37 and 38, we have

$$
\begin{aligned}
m \pi c_{2}=\frac{1}{2} A_{n}\left(k_{n} k_{w}+n^{2} \pi^{2}\right) & \exp \left(-i \sigma x_{1}\right), \\
\left(m \pi c_{2}-k_{m} A_{m}^{\prime}\right) \cos m \pi= & \frac{1}{2} A_{n}\left(k_{n} k_{w}+n^{2} \pi^{2}\right) \\
& \times \cos n \pi \exp \left[-i\left(\sigma x_{1}+\theta\right)\right] .
\end{aligned}
$$

Solving Eq. 43 for $c_{2}$ and substituting the result into Eq. 44, we obtain the following solvability condition for Eqs. 36-38:

$A_{m}^{\prime}=\frac{1}{2} k_{m}^{-1}\left(k_{n} k_{w}+n^{2} \pi^{2}\right)\left[1-(-1)^{m+n} \exp (-i \theta)\right] A_{n} \exp \left(-i \sigma x_{1}\right)$.

Similarly, the solvability condition for Eqs. 39-41 is

$A_{n}^{\prime}=\frac{1}{2} k_{n}^{-1}\left(k_{m} k_{w}-m^{2} \pi^{2}\right)\left[1-(-1)^{m+n} \exp (i \theta)\right] A_{m} \exp \left(i \sigma x_{1}\right)$.

We seek a solution to Eqs. 45 and 46 of the form

$$
A_{m}=a_{m} \exp \left(s x_{1}\right), A_{n}=a_{n} \exp \left[(s+i \sigma) x_{1}\right],
$$

where $a_{j}$ and $s$ are constants. Substituting this assumed solution into Eqs. 45 and 46 and eliminating the $a$ 's, we get

$$
s(s+i \sigma)=\Omega,
$$

where

$\Omega=\frac{1}{2}\left(k_{m} k_{n}\right)^{-1}\left(k_{m} k_{w}-m^{2} \pi^{2}\right)\left(k_{n} k_{w}+n^{2} \pi^{2}\right)\left[1-(-1)^{m+n} \cos \theta\right]$.

The solution of Eq. $48 \mathrm{a}$ is

$$
s=\frac{1}{2} i\left[-\sigma \pm\left(\sigma^{2}-4 \Omega\right)^{1 / 2}\right] .
$$

Since $k_{w}=k_{n}-k_{m}+\epsilon \sigma$ from Eq. 33,

$$
k_{m} k_{w}-m^{2} \pi^{2}=k_{n} k_{m}-k_{m}^{2}+\epsilon \sigma k_{m}-m^{2} \pi^{2}=k_{n} k_{m}-\omega^{2}+\epsilon \sigma k_{m}<0,
$$

because $k_{n}<\omega$ and $k_{m}<\omega$. Hence $s$ is pure imaginary and $A_{m}$ and $A_{n}$ are bounded as a consequence. Therefore, $\phi_{0}$ and $\phi_{1}$ are bounded according to Eqs. 29, 42; and
43, and the response is not very large in contrast with the straightforward expansion obtained in the previous section. However, Eqs. 29, 45, and 46 show that the $n$th mode cannot exist without the $m$ th resonant mode. Consequently, sinusoidal wall undulations can be used to generate the $m$ th mode from the $n$th mode if $k_{m} \approx k_{n}-k_{w}$.

\section{CONCLUDING REMARKS}

A straightforward perturbation solution of the form $\phi=\phi_{0}+\epsilon \phi_{1}$ is obtained for the acoustic wave propagation in a hard-walled two-dimensional duct whose walls have weak sinusoidal undulations of the order of the small dimensionless parameter $\epsilon$. The results show that if the $m$ th mode corresponding to the frequency $\omega$ with the wavenumber $k_{m}$ passes through the duct, the wall undulations will generate two weak waves with the wavenumbers $k_{w}+k_{m}$ and $k_{w}-k_{m}$, where $k_{w}$ is the wavenumber of the wall undulations. If $k_{w} \mp k_{m} \approx k_{n}$ where $k_{n}$ is the wavenumber of the $n$th mode, the straightforward expansion breaks down because $\epsilon \phi_{1}$ is not small compared with $\phi_{0}$. For traveling waves, only the resonant case $k_{w} \approx k_{n}-k_{m}$ occurs. An expansion valid when $k_{w} \approx k_{m}-k_{n}$ is then obtained. The results show that the $m$ th mode cannot exist without strongly generating the $n$th mode; however, both modes travel unattenuated through the duct.

\section{ACKNOWLEDGMENTS}

The author is grateful to Mr. O. R. Asfar for helpful comments and discussions and to Dr. J. E. Kaiser for reading the manuscript. This work was supported by the NASA Langley Research Center under Grant NGR47-004-109.

${ }^{1}$ M. A. Isakovitch, Akust. Z. 3, 37-45 (1957).

${ }^{2}$ J. S. Samuels, J. Acoust. Soc. Am. 31, 319-325 (1959).

${ }^{3}$ R. F. Salant, J. Acoust. Soc. Am. 53, 504-507 (1973).

${ }^{4}$ A. H. Nayfeh, Perturbation Methods (Wiley-Interscience, New York, 1973), Chap. 6. 